\title{
The development of a questionnaire to assess the attitudes of older people to end-of-life issues (AEOLI)
}

\author{
Susan Catt, Martin Blanchard Department of Mental Health Sciences, University College, London, \\ Julia Addington-Hall School of Nursing and Midwifery, University of Southampton, Southampton, Maria Zis \\ Department of Palliative Care and Policy, Kings College, London and Bob Blizard, Michael King Department of \\ Mental Health Sciences, University College, London
}

\begin{abstract}
Objectives: To develop an end-of-life attitudes questionnaire for use in a large communitybased sample of older people. Design: Nominal groups and standardization of questions. Participants: Eighteen older people, ten academics and five specialist palliative care health professionals were involved in nominal groups. Thirty older people took part in initial pilot work and a further 50 were involved in reliability testing. Results: A 27-item attitudes of older people to end-of-life issues (AEOLI) questionnaire. Discussion: In modern times, death and dying predominantly occurs among older people and yet we know very little about older people's attitudes to end-of-life care. The AEOLI questionnaire can be used in large scale surveys to elicit attitudes on end-of life issues considered important by older people and health care professionals. Palliative Medicine 2005; 19: 397-401
\end{abstract}

Key words: aged; attitude measures; death and dying

\section{Introduction}

Cancer patients in the UK who receive care from hospice and specialist palliative care services are younger than those who do not receive this service. ${ }^{1-4}$ The reasons for this are poorly understood. In common with the relative neglect of older people in health services research, few studies have investigated the relationship between patient age and use of hospice and specialist palliative care services, and few studies have asked older people what sort of care they would prefer if they were to become terminally ill.

It may be appropriate for specialist palliative care providers to concentrate their limited resources on younger patients if, as has been suggested, older patients are more accepting of death and hence need less psychological and spiritual support, or if they experience less severe symptoms and distress. It may also be the case that older people themselves do not want the types of care provided under the hospice philosophy and by specialist palliative care teams. However, little is known about the attitudes of older people to these and other important end-of-life issues that have substantial policy implications. Better information on how older people view these topics and on the determinants of their views, will help ensure that service developments in hospice and specialist palliative care are acceptable to service users and lead to care that is relevant to the needs and wishes of patients.

Address for correspondence: Martin Blanchard, Department of Mental Health Sciences, Royal Free and University College Medical School, University College, London, UK.

E-mail:m.blanchard@medsch.ucl.ac.uk
This paper describes the development of a questionnaire designed for use in a large, community-based survey of older people; the attitudes of older people to end-of-life issues (AEOLI) questionnaire, its structure, content and standardization.

\section{Methods}

\section{Literature review}

The major databases, Index Medicus/MEDLINE, EMBASE, Psychinfo and CINAHL were systematically searched with key search words for relevant papers and measures, which had already been developed to assess older people's attitudes to the end-of-life. The instruments found to be available were too narrow in scope for our purposes, often only addressing a single issue. Examples included measures such as the Preferences for Care near the End of Life scale (PCEOL), ${ }^{5}$ or the Domino scale, a 12-item psychometric instrument, which measures attitudes toward physician-assisted suicide (PAS). ${ }^{6}$ The use of such measures would have required any survey to cover end-of-life issues in a piecemeal fashion, potentially employing a large number of separate instruments (with many questions each). Also, few of these instruments had been widely used in the general older population. The solution to these dilemmas was to develop a tool for our needs.

\section{General conception}

Our aim was to produce an instrument on end-of-life issues for use in a large, quantitative survey. A brief questionnaire needed to be developed that would take 
Table 1 Items generated by nominal group sessions with older people

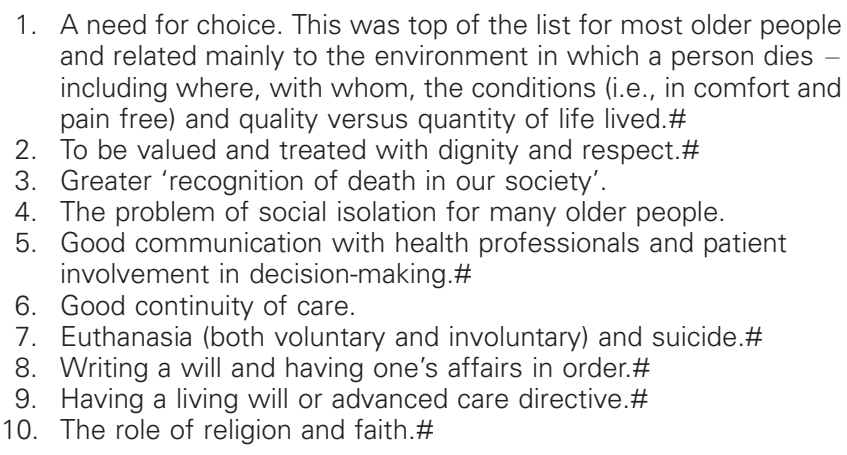

Items marked \# were also suggested by the academics and palliative care professionals.

account of the sensitivity of the subject, would be appropriate to an older age group and would be clear and unambiguous in its wording. The construction of an interview-based rather than a self-report measure was chosen because of the nature of the questions being asked, the possible need to support participants during questioning and the possible need to facilitate completion in the very elderly.

\section{The population}

The older people who helped us to develop the questionnaire were volunteers drawn from local general practices, recruited when they visited their practice or by letter. Because of the nature of the topic, we worked closely with the general practitioners in our decisions as to whom to approach.

\section{Generation of topics to be surveyed}

In establishing which topics should be covered, it was necessary to strike a balance between reviews of the scientific literature and the issues that concerned older people, palliative care professionals and academics in this

Table 2 Additional items generated by the academics and palliative care professional nominal group sessions but not mentioned by the older people

1. Emergency resuscitation

2. Costs of care - private and public care

3. The attitudes of others - ageism

4. Prevention - promoting health/survival

5. The process of dying, fear of dying rather than death, worries about organising for death, readiness for death

6. The loss of a future

7. Fear of drugs

8. Sexuality, losing sense of self, of male or femaleness

9. Safety and support

10. Letting others down

11. Positive natural process

12. Not wanting to be a burden

13. Loss of status and income (in younger people) area. Thus, a series of nominal groups were conducted with older people and palliative care professionals.

\section{Nominal group technique}

Nominal groups are a form of focus group that enables all members of the group initially to voice opinions on a topic and then, through the group process, rank in importance the consensus views discussed. ${ }^{7}$ Group sessions are brought together under the guidance of a skilled group leader and a facilitator. The optimum discussion group size is between five and eight people. We asked participants to discuss issues related to cancer, death, dying and palliative care. They were then asked to prioritize the issues they considered the most important for older people in relation to health care near the end-oflife. The groups were audio-taped so that the discussion could be cross checked against the written records produced during the nominal process.

Three nominal groups were carried out with older people. Grouping was by age $(55-64$ years $(n=4) ; 65-74$ years $(n=6) ;>75(n=8))$ with a mix of men and women in each group. Participants were volunteers from two general practices local to the Royal Free Hospital in North London (one in an affluent area, the other in a socio-economically deprived area). Two nominal groups were conducted with a group of social science academics $(n=10)$ and a group of specialist palliative care professionals $(n=5)$.

\section{Development of attitude questions}

The nominal group material was used to develop questions designed to elicit attitudes towards a broad range of issues in end-of-life care.

\section{Standardization of questions}

\section{Comprehensibility and face-validity}

Forty-seven questions were generated and piloted with 30 older volunteers from two general practices, who were asked to comment on their appropriateness, readability and comprehensibility. The wording was adjusted accordingly.

\section{Reliability}

We asked an additional group of 50 older volunteers from general practices to complete the penultimate version of the questionnaire on two occasions, two weeks apart. These data were subjected to test-retest reliability. Two indices were adopted to evaluate the reliability of the items and scales. The test-retest reliability was assessed using Cohen's Kappa statistic for categorical variables. Kappa values $>0.40$ are considered to represent moderate concordance, values $>0.6$ reflect substantial concordance. We rejected item Kappa values $<0.40$, leaving 


\section{Decision making}

Attitude 1:

If I were severely ill with no hope of recovery, I would prefer the doctors to make all the decisions about my care.

Pain

Attitude 5:

If I were severely ill with no hope of recovery, I would want treatment to control my pain completely, even if it left me muddled.

Attitude 9:

If I were severely ill with no hope of recovery, I would like to be in control of my pain medication rather than relying on doctors and nurses to give it to me.

Attitude 20:

I believe most people are in pain as death approaches.

\section{Care environment}

Attitude 3:

If I were severely ill with no hope of recovery, I would like to die alone.

Attitude 6:

If I were severely ill with no hope of recovery, I would rather be cared for in a hospice than at home.

Attitude 10:

If I were severely ill with no hope of recovery, I would rather be cared for in a hospital than a hospice.

Attitude 15:

If I were severely ill with no hope of recovery, I would rather be cared for in hospital than at home.

Attitude 27:

If I were severely ill with no hope of recovery, it would not matter how many health professionals I saw as long as I was getting the latest treatment.

\section{Living wills}

Attitude 8:

By law, doctors should have to follow the instructions in a living will.

\section{Attitude 14:}

If I were severely ill with no hope of recovery, I would not need a living will as my loved ones would know what to do when the time comes.

Attitude 19:

If I were severely ill with no hope of recovery, I would like a living will so that the doctors and nurses knew exactly what care I wanted.

Attitude 24:

I find the idea of making a living will too gloomy.

\section{Euthanasia/Physician assisted suicide}

Attitude 4:

If I were severely ill with no hope of recovery, I would like a drug at my disposal to end my life when I chose.

Attitude 17:

A person should never take their own life.

Attitude 18:

If my loved ones thought it better for me to die because I was severely ill with no hope of recovery, doctors should be allowed to help me to die.

Attitude 26:

If I were severely ill with no hope of recovery, my doctor should be allowed to help me to end my life.

Figure 1 Statements in the final version of the questionnaire. 


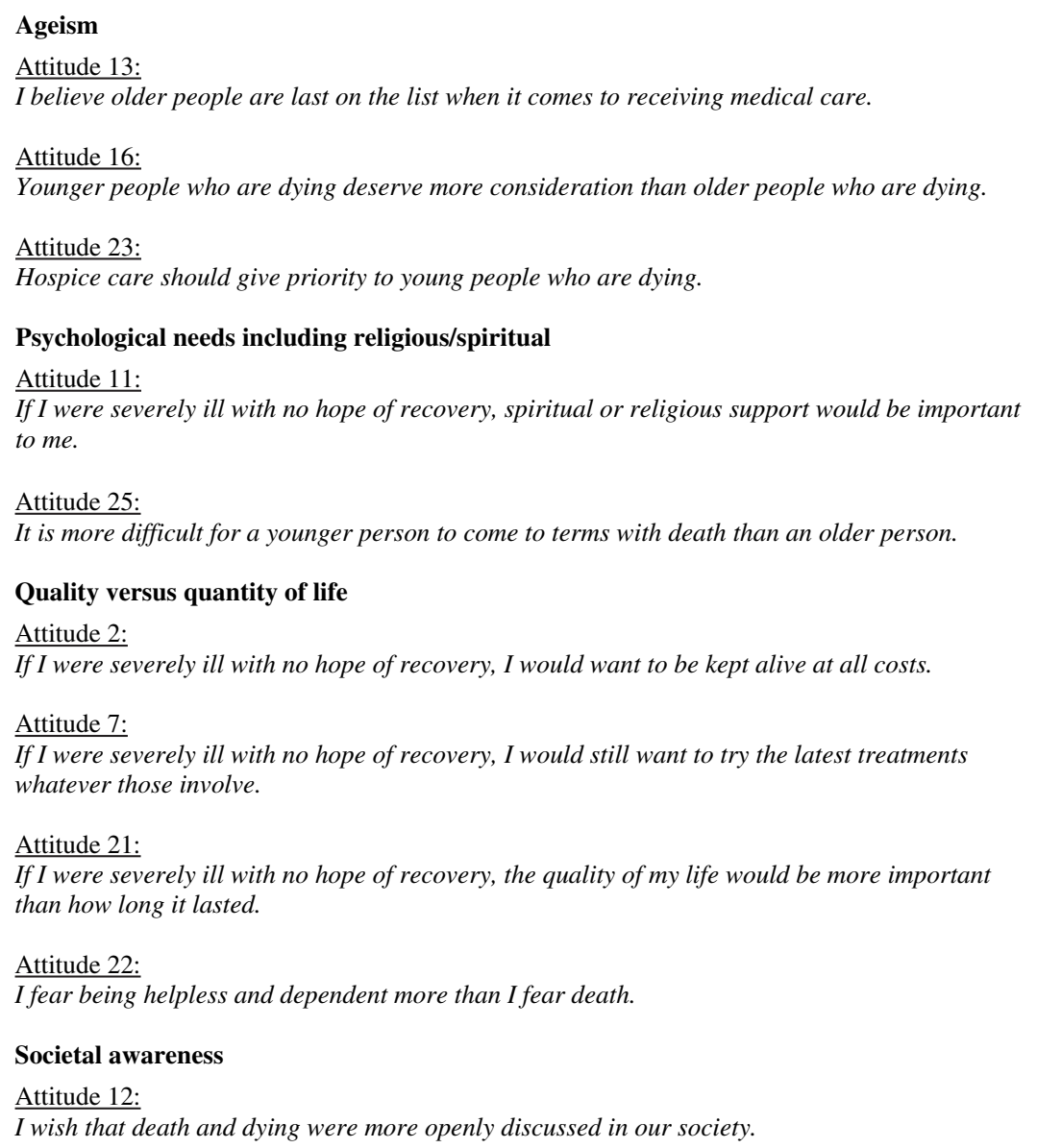

Figure 1 (Continued)

a final set of 27 questions. The question of internal consistency was assessed using Cronbach's Alpha statistic. This assumes that there is an underlying true score; Cronbach's Alpha measures the proportion of the variability in a scale that is due to the true score (i.e., that which is not due to error).

\section{Other pilot work}

A number of different scale formats were tested for recording participants' responses to the attitude questions. The groups of patients who participated in the nominal group work were presented with a variety of scale formats and were asked to provide feedback on their usability. Formats tested included, for example, Likert scales versus a line scale (visual analogue scale). The number of response options, e.g., 1-4, 1-5, 1-7, was explored together with scale labelling, including wording.

\section{Ethical issues}

Ethical approval was obtained from the North Thames Multisite Regional Ethical Committee and the Local Research Ethical Committees involved.

\section{Results}

\section{Nominal group work}

From the nominal group work, the topics shown in Table 1 were considered by older people as most important when addressing the issue of health care needs at the end-of-life. The items marked with \# were also produced by the nominal group sessions held with the social science academics and palliative care workers. Further issues raised by them are included in Table 2.

The final standardized questions cover the areas shown in Figure 1.

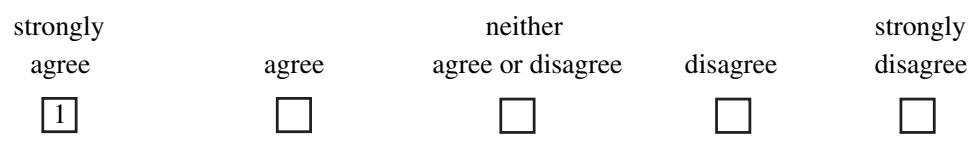

Figure 2 Example of the scale used with attitude questions. 


\section{Response scale format}

Following participants' feedback in the pilot work, it was concluded that a five-point Likert scale with labels for each available response, ranging from 'strongly agree' to 'strongly disagree' (Figure 2) was the simplest and most appropriate scale to accompany the attitude questions, particularly for the oldest participants in our population.

\section{Internal consistency of themes}

Not all the areas covered in the questionnaire are open to testing of internal consistency, as questions cover different aspects of a theme. However, there is evidence of internal consistency (an underlying true score) in 'living wills' (Cronbach alpha $=0.68$ ), 'euthanasia and physician-assisted suicide' (Cronbach alpha $=0.77$ ) and 'quality versus quantity of life' (Cronbach alpha $=0.52$ ).

\section{Discussion}

The attitudes of older people to end-of-life issues (AEOLI) questionnaire is a 27-item interview designed to elicit attitudes to important end-of-life issues in largescale quantitative surveys. Its strength is that the items it contains were generated using nominal group sessions with older people as well as academics and clinical health professionals. The questions used were carefully developed with regard to comprehensibility and face validity as assessed by older people, as was the response scale format. A test-retest reliability study using older people provided us with questions with reasonable Kappa statistics, and there is some evidence of good internal consistency.

Other workers have used focus groups to explore older people's views and values about advanced care statements, ${ }^{8}$ and innovative technologies. ${ }^{9}$ A further research group has used focus groups and semi-structured interviews $(n=45)$ to explore the attitudes of older people towards home as a place of care when dying. ${ }^{10}$ No studies appear to have used a methodology as presented in this paper to develop a standardized questionnaire.

The AEOLI should provide reliable and valid information on the attitudes of older people to death, palliative treatment and hospice care that could prove useful in the development of future services. It will also enable researchers to explore differences in attitudes between older populations and their correlates. The AEOLI therefore has the potential to make an important contribution to extending knowledge in this underresearched area.

\section{Acknowledgements}

We would like to thank all the patients and general practice staff who took part in the study. This work was undertaken by the authors who received funding from the NHS London Regional Office, Research and Development, Responsive Funding Programme. The views expressed in the publication are those of the authors and not necessarily those of the NHS or the Department of Health.

\section{References}

1 Addington-Hall JM, Altmann D, McCarthy M. Who gets hospice in-patient care? Soc Sci Med 1998; 46: 1011-16.

2 Addington-Hall J, Altmann D. Which terminally ill cancer patients in the United Kingdom receive care from community specialist palliative care nurses? $J A d v$ Nurs 2000; 32: 799-806.

3 Gray J, Foster D. Factors associated with utilization of specialist palliative care services: a population based study. J Pub Health Med 1997; 19: 464-69.

4 Burge F, Johnstone G, Lawson B et al. Population-based trends in referral of the elderly to a comprehensive palliative care programme. Palliat Med 2002; 16: 255 56.

5 Gauthier DM, Froman RD. Preference for care near the end of life: scale development and validation. Res Nurs Health 2001; 24: 298-306.

6 Domino G. Community attitudes toward physician assisted suicide. Omega- J Death Dying 2002; 46: 199214.

7 Delbeq AL, Van den Ven AH, Gustafson DH. Group techniques for programme planning. A guide to nominal group and Delphi processes. Glenview, IL: Scott, Foresman and Company, 1975.

8 Seymour J, Gott M, Bellamy G, Ahmedzai SH, Clark D. Planning for the end of life: the views of older people about advance care statements. Soc Sci Med 2004; 59: 57-68.

9 Seymour J. Technology and 'natural death': a study of older people. Z Gerontol Geriat 2003; 36: 339-46.

10 Gott M, Seymour J, Bellamy G, Clark D, Ahmedzai S. Older people's views about home as a place of care at the end of life. Palliat Med 2004; 18: 460-67. 